\title{
蝶蚂催化抗凝血药华法林及其衍生物的合成
}

\author{
李志林 ${ }^{a}$ 周海燕 $b \quad$ 官 智 $*, a$ \\ ( ${ }^{a}$ 西南大学化学化工学院 重庆市应用化学重点实验室 重庆 400715) \\ $\left({ }^{b}\right.$ 西南大学生命科学学院 重庆 400715)
}

\begin{abstract}
摘要 报道了蚯蚂粗提物作为生物催化剂催化华法林及其衍生物的合成. 采用 4-差基香豆素与苄叉丙酮作为原料, 探 究了溶剂、催化剂的量、含水量、投料比、温度、反应时间对 Michael 反应合成华法林的影响. 获得最佳反应条件为 $10 \%$ 含水量, $100 \mathrm{mg}$ 虹蚓粗提物, $50{ }^{\circ} \mathrm{C}$ 下反应 $48 \mathrm{~h}, 4$-羊基香豆素与苄叉丙酮投料比为 $1: 3$ 时华法林的最高收率为 98\%, 其衍生物的收率为 57\% 99\%, 并有一定的对映选择性(达 $20 \% e e$ ). 对照实验表明虹蚂粗提物中的酶催化了该反 应. 这项工作提供了一个很好的利用天然催化剂合成药物分子及其衍生物的例子.
\end{abstract}

关键词 生物催化; 虾蚓粗提物; 华法林; Michael 加成

\section{Synthesis of Anticogulant Warfarin and Its Derivatives by the Crude Earthwarm Extract}

\author{
Li, Zhilin ${ }^{a} \quad$ Zhou, Haiyan $^{b} \quad$ Guan, Zhi* ${ }^{*, a}$ \\ ( ${ }^{a}$ Key Laboratory of Applied Chemistry of Chongqing Municipality, School of Chemistry and Chemical Engineering, \\ Southwest University, Chongqing 400715) \\ ( ${ }^{b}$ School of Life Sciences, Southwest University, Chongqing 400715)
}

\begin{abstract}
This article describes the synthesis of warfarin and its derivatives by using the crude earthwarm extract as a biocatalyst. Warfarin, an effective anticogulant being used for half a century, has enormous clinic and commercial value. Warfarin and its derivatives are usually prepared through chemical catalysis while biocatalysis was rarely explored. Because biocatalysis is green, sustainable and usually under mild conditions, it is necessary to develop biocatalytic methods for these compounds. It is found that the crude extract of earthwarms is a fine biocatalyst. Earthwarms are harmless and abundant in nature. There are many hydrolases in the alimentary tract of earthwarms, and among of them, proteases are most thoroughly studied. The crude earthwarm extract from Eisenia foetida was prepared, and it was used as a cheap and ecologically friendly biocatalyst for the synthesis of warfarin and its derivatives. The procedure is simple and no additional cofactors and equipments are required. The best results were obtained using $100 \mathrm{mg}$ of earthworm extract in DMSO/water $(10 \%$ water, $V / V)$ with $1: 3$ molar ratio of 4-hydroxycoumarin to benzylideneacetone at $50{ }^{\circ} \mathrm{C}$ for $48 \mathrm{~h}$. Warfarin was obtained in an excellent yield of $98 \%$, and its derivatives were achieved in yields of $57 \% \sim 99 \%$ with some enantioselectivities (up to $20 \%$ ee). In addition, control experiments demonstrated that enzyme(s) in the crude extract of earthwarms catalyzed Michael addition reaction. This work provides an alternative method for the synthesis of warfarin and its derivatives by using an easily available natural catalyst.
\end{abstract}

Keywords biocatalysis; the crude extract of earthworms; warfarin; Michael reaction

华法林, 作为维生素 $\mathrm{K}$ 的拮抗剂, 是目前唯一广泛 应用于临床、口服吸收效果最好的抗凝血药物, 特别适 用于抗凝患者的长期治疗 ${ }^{[1,2]}$. 华法林的传统合成是以 三乙胺、吡啶、喹啉等碱性物质为催化剂, 4-差基香豆 素和芐叉丙酮为底物经 Michael 加成获得. 其中, 手性
伯胺或经修饰改进的伯胺 ${ }^{[3 \sim 8]}$ 作为催化剂可获得理想的 收率及较好的对映选择性，但这些基于味唑烷酮 ${ }^{[0]}$ 、生 物碱、氨基酸衍生物和其他手性物质的有机催化剂相当 昂贵 ${ }^{[10]}$. 除了以上有机试剂催化方法，还有人采用金属 或金属与有机双催化剂，或者使用手性助剂 ${ }^{[11]}$. 可是,

\footnotetext{
* Corresponding author. E-mail: guanzhi@swu.edu.cn

Received December 11, 2016; revised January 11, 2017; published online February 8, 2017.

Project supported by the National Training Program of Innovation and Entrepreneurship for Undergraduates (No. 201510635004).

“国家级大学生创新创业训练计划” 基金(No. 201510635004)资助项目.
} 
有机金属催化剂却隐藏着有毒金属产物的污染等问题. 而且，金属或者有机催化剂，常常具备以下诸多缺点: 多步反应、消耗不可再生的原材料 ${ }^{[11]}$ (包括一些有毒物 质和昂贵的材料). 因此, 化学家们致力于研究获得廉 价、环境友好型的绿色催化剂.

随着绿色化学研究的推进, 我们对酶用于有机合成 的研究越来越深入. 酶作为一种生物可再生的、生物可 降解的无环境毒害的资源 ${ }^{[9]}$, 凭借其各种适宜于化学催 化的特性, 得到了生化学家们的青睐. 据我们了解, 到 目前为止, 关于酶催化合成华法林的研究有三篇报道. Sano 等 ${ }^{[11]}$ 用脂肪酶 $\mathrm{AS}$ 催化, 得到 $85 \%$ 的收率及 $45 \%$ ee. Liu 等 ${ }^{[12]}$ 将脂肪酶作为基质, 金属有机材料作为酶的骨 架(MOFs), 催化 Michael 反应合成华法林，得到了 $87 \%$ 的收率, 但是没有对映选择性. 我们小组 ${ }^{[13]}$ 用猪胰脂肪 酶(PPL)催化合成华法林，得到 $94 \%$ 的收率及 $28 \% e e$. 以上所用的酶都是经纯化提取的.

酶的粗提物作为一种新型的生物催化剂, 相比于分 离纯化困难、耗时、昂贵的纯酶, 具备便宜易获得的特 点, 并且避免了在酶的纯化过程中使用的对环境造成危 害的试剂和药品, 更符合环境友好的要求 ${ }^{[14,15]}$. 我们发 现, 蚯蚓粗提物是一种很好的生物催化剂. 蝶蚓是自然 界中分布较广的无害生物, 在自然界中扮演分解者的角 色. 蚳蚓消化道有多种水解酶, 其中蛋白酶是目前研究 最透彻的. 据报道, 粉正蚓中丝氨酸蛋白酶是由 6 种同 工酶构成, 具溶解纤维蛋白的活性 ${ }^{[16]}$. 常文瑞院士团 队 ${ }^{[17]}$ 分离并鉴定出 7 种蝶蚓纤溶酶的活性组分(组分 $\mathrm{A}$ 为丝氨酸蛋白酶). 由此可知, 蚳蚓中存在一系列结构 不同的蛋白酶. 我们用赤子爱胜蚓(Eisenia foetida)来制 作蚳蚓粗提物, 并且发现蚯蚓粗提物可用于催化不对称 Mannich、aldol、Henry、Biginelli 反应, 以及三组分的 aza-Diels-Alder (Mannich-Michael)反应合成异喹啉及其 衍生物, 还可以催化 domino 反应合成香豆素 ${ }^{[14,15]}$. 可 见, 蚳蚓粗提物作为简单易得的绿色催化剂, 应用前景 广阔。

因此, 我们选择蚯蚓粗提物这一天然存在的生物催 化剂用于抗凝血药华法林的合成, 并获得了最优催化条 件: 含水量为 $10 \%$, 蚳蚓粗提物 $100 \mathrm{mg}, 50{ }^{\circ} \mathrm{C}$ 下反应 48 $\mathrm{h}, 4$-着基香豆素与苄叉丙酮投料比为 $1: 3$ 时华法林的 最高收率为 $98 \%$, 其衍生物的收率为 $57 \% \sim 99 \%$, 并具 有一定的对映选择性(达 $20 \% e e$ ).

\section{1 结果与讨论}

我们采用之前报道的方法来制备蚳蚓粗提物 ${ }^{[14]}$, 用新鲜的赤子爱胜蚓(Eisenia foetida) 和去离子水制成匀 浆, 通过蔗糖透析浓缩, 再离心获得上清液, 干燥后研
磨成粉末, 即可获得蚳蚓粗提物. 我们选用 4-着基香豆 素和茮叉丙酮作为模型反应(Eq. 1), 蝶蚂粗提物作为催 化剂，进行反应条件的优化.

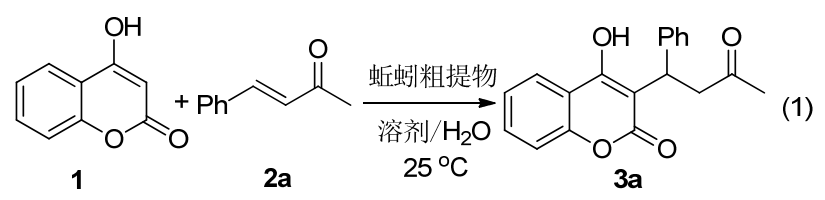

首先进行了溶剂的篎选. 二甲亚砜作为溶剂时得到 高达 $87 \%$ 的收率及 $20 \% e e($ 表 1, Entry 1), 远远高出所篮 选的其他溶剂的收率. 水作为溶剂时仅得到 $3 \%$ 的收率 和 $3 \%$ 的 $e e$ 值(表 1, 序号 10). 因此, 我们选择了二甲亚 砜作为该反应的最佳溶剂. 在所有笁选的溶剂里, 蚳蚓 粗提物呈悬浮状，是一个非均相的反应体系.

表 1 溶剂对蚯蚓粗提物催化 Michael 反应的影响 ${ }^{a}$ Table 1 Influence of solvents on the crude earthwarm extract catalyzed-Michael reaction

\begin{tabular}{clcc}
\hline Entry & \multicolumn{1}{c}{ Solvent } & $\mathrm{Yield}^{b} / \%$ & $e e^{c} / \%$ \\
\hline 1 & 二甲亚砜 & 87 & 20 \\
2 & $N, N$-二甲基甲酰胺 & 47 & 11 \\
3 & 乙酸乙酯 & 33 & 3 \\
4 & 甲基叔丁基醚 & 33 & 3 \\
5 & 乙腈 & 30 & 0 \\
6 & 1,4-二氧六环 & 28 & 0 \\
7 & 二氯甲烷 & 27 & 0 \\
8 & 无水乙醇 & 22 & 3 \\
9 & 环己烷 & 12 & 5 \\
10 & 水 & 3 & 3 \\
\hline
\end{tabular}

${ }^{a}$ 反应条件: 1 ( $\left.0.5 \mathrm{mmol}\right), \mathbf{2 a}(2.5 \mathrm{mmol}, 5$ equiv.), 蝶蚂粗提物 $(100 \mathrm{mg})$, 溶 剂 $(0.90 \mathrm{~mL})$ 和去离子水 $(0.10 \mathrm{~mL})$, 在 $25{ }^{\circ} \mathrm{C}$ 下反应 $120 \mathrm{~h} ;{ }^{b}$ 收率是通过硅 胶色谱柱分离得到; ${ }^{c} e e$ 是通过手性高效液相色谱(Chiralpak AD-H 手性 柱)分析得到.

接着, 探究了蚳蚓粗提物的量对华法林合成的影 响. 随着蚳蚓粗提物量的增加, 收率和对映选择性也有 所增加(表 2, Entries 1 5). 蚳蚓粗提物的量为 100 和 $110 \mathrm{mg}$ 时, 收率最高, 为 $87 \%$ (表 2 , Entries 10 和 11). 但 当蚳蚓粗提物的量超过 $110 \mathrm{mg}$ 时, 收率减少(表 2, Entries 12 和 13). 原因可能是蚯蚓粗提物在溶剂中呈悬浮 状, 过量会影响反应的进行. 因此, 选择 $100 \mathrm{mg}$ 蚯蚓粗 提物为最佳用量.

探讨了含水量对蚯蚓粗提物催化合成华法林反应 的影响. 体系中含水量对收率和对映选择性均有明显影 响. 随着含水量的增加, 收率有所增加, ee 也有所增加 (表 3, Entryies 1 3). 当含水量为 $10 \%$ 的时候, 得到最 高产率 $87 \%$ 及 $20 \% e e($ 表 3, Entry 4). 但是之后随着含水 量的增加, 收率和 $e e$ 明显下降, 可能是在含水量高的体 系中, 底物的溶解性下降造成的. 因此选择 $10 \%$ 的含水 量作为最优反应条件. 
表 2 蚯蚓粗提物的量对 Michael 反应的影响 ${ }^{a}$

Table 2 Effects of amount of crude earthwarm extract on the Michael reaction ${ }^{a}$

\begin{tabular}{cccc}
\hline Entry & 蚯蚓粗提物 $/ \mathrm{mg}$ & $\mathrm{Yield}^{b} / \%$ & $e e^{c} / \%$ \\
\hline 1 & 10 & 17 & 2 \\
2 & 20 & 27 & 6 \\
3 & 30 & 37 & 12 \\
4 & 40 & 56 & 14 \\
5 & 50 & 68 & 22 \\
6 & 60 & 75 & 20 \\
7 & 70 & 80 & 19 \\
8 & 80 & 85 & 19 \\
9 & 90 & 86 & 20 \\
10 & 100 & 87 & 20 \\
11 & 110 & 87 & 19 \\
12 & 120 & 75 & 19 \\
13 & 140 & 72 & 19 \\
\hline
\end{tabular}

${ }^{a}$ 反应条件: 1 ( $\left.0.5 \mathrm{mmol}\right), \mathbf{2 a}(2.5 \mathrm{mmol}, 5$ equiv.), 蝶蚂粗提物作为催化剂, 二甲亚砜 $(0.90 \mathrm{~mL})$ 和去离子水 $(0.10 \mathrm{~mL})$, 在 $25{ }^{\circ} \mathrm{C}$ 下反应 $120 \mathrm{~h} ;{ }^{b}$ 收率是 通过硅胶色谱柱分离得到; ${ }^{c} e e$ 是通过手性高效液相色谱(Chiralpak AD-H 手性柱)分析得到.

表 3 含水量对蚯蚓粗提物催化的 Michael 反应的影响 ${ }^{a}$

Table 3 Effects of water content on the crude earthwarm extract catalyzed-Michael reaction

\begin{tabular}{cccc}
\hline Entry & Water content & Yield $^{b} / \%$ & $e e^{c} / \%$ \\
\hline 1 & 0 & 84 & 21 \\
2 & 5 & 85 & 20 \\
3 & 10 & 87 & 20 \\
4 & 15 & 67 & 14 \\
5 & 20 & 63 & 12 \\
6 & 30 & 48 & 5 \\
7 & 40 & 42 & 3 \\
8 & 50 & 26 & 0 \\
\hline
\end{tabular}

${ }^{a}$ 反应条件: 1 ( $\left.0.5 \mathrm{mmol}\right), \mathbf{2 a}(2.5 \mathrm{mmol}, 5$ equiv. $)$, 虹蚂粗提物 $(100 \mathrm{mg})$, 溶 剂(二甲亚砜和去离子水总体积 $1.0 \mathrm{~mL}$ ), 在 $25{ }^{\circ} \mathrm{C}$ 下反应 $120 \mathrm{~h} ;{ }^{b}$ 收率是通 过硅胶色谱柱分离得到; ${ }^{c} e e$ 是通过手性高效液相色谱(Chiralpak AD-H 手 性柱)分析得到.

接着探讨了投料比对反应的影响. 当投料比 $1: 2 \mathrm{a}$ 为 $1: 1$ 时, 收率仅为 $59 \%, e e$ 为 $22 \%$ (表 4, Entry 1), 增 加投料比至 1:3, 收率提高到 $87 \%, e e$ 无明显变化(表 4, Entry 3). 继续增加投料比, 收率变化不大, 考虑到收率 和原料的经济性, 我们最终选择 $1: 3(1: 2 a)$ 作为最佳 投料比.

温度会影响酶的生物活性和稳定性, 因此温度在酶 催化的反应中也是一个重要的因素. 每一种酶对温度的 敏感程度和最适催化温度都不同, 有的耐高温, 有的能 耐受其他极端环境. 接下来我们探究了温度对蚯蚂粗提 物催化的 Michael 反应的影响. 由表 5 可知, 从 15 到 $50{ }^{\circ} \mathrm{C}$, 该反应收率随温度升高而有所增加. 温度升高 到 $50{ }^{\circ} \mathrm{C}$ 时, 可获得最高收率 $97 \%$ (表 5, Entry 6). 但与 之相反的是, ee 值随温度升高呈下降趋势. 但温度升高 到 $60{ }^{\circ} \mathrm{C}$ 时, 收率有所下降. 可能是温度过高而造成酶
表 4 底物投料比对蚯蚓粗提物催化的 Michael 反应的影响 ${ }^{a}$ Table 4 Influence of mole ratio of substrates on the crude earthwarm extract catalyzed-Michael reaction ${ }^{a}$

\begin{tabular}{cccc}
\hline Entry & $\mathbf{1}: \mathbf{2 a}$ & Yield $^{b} / \%$ & $e e^{c} / \%$ \\
\hline 1 & $1: 1$ & 59 & 22 \\
2 & $1: 2$ & 74 & 22 \\
3 & $1: 3$ & 87 & 20 \\
4 & $1: 4$ & 88 & 18 \\
5 & $1: 5$ & 87 & 20 \\
\hline
\end{tabular}

${ }^{a}$ 反应条件: 虹蚂粗提物 $(100 \mathrm{mg})$, 二甲亚砜 $(0.90 \mathrm{~mL})$ 和去离子水 $(0.10$ $\mathrm{mL}$ ), 在 $25{ }^{\circ} \mathrm{C}$ 下反应 $120 \mathrm{~h} ;{ }^{b}$ 收率是通过硅胶色谱柱分离得到; ${ }^{c} e e$ 是通 过手性高效液相色谱(Chiralpak AD-H 手性柱)分析得到.

的活力下降所致. 在 25 和 $60{ }^{\circ} \mathrm{C}$ 之间收率相差不大, 说 明我们使用的虹蚓提取物中的酶在这个温度下能保持 稳定. Nakajima 等 ${ }^{[16]}$ 报道了粉正蚓中的碱性丝氨酸蛋白 酶在 $60{ }^{\circ} \mathrm{C}$ 以下, $\mathrm{pH} 2 \sim 11$ 条件下都能保持稳定性, 并 且对有机溶剂和去垢剂都有较好的耐受性，在室温条件 下储存多年均保持其活性; Zhao 等 ${ }^{[18]}$ 的研究表明, 蝶蚓 纤溶酶是一种热稳定性非常好的蛋白水解酶, 在 $50{ }^{\circ} \mathrm{C}$ 以下可以长期保持活性. 所以, 最终选择 $50{ }^{\circ} \mathrm{C}$ 作为最 佳反应温度.

表 5 温度对蚯蚓粗提物催化的 Michael 反应的影响 ${ }^{a}$

Table 5 Effects of temperature on the crude earthwarm extract catalyzed-Michael reaction ${ }^{a}$

\begin{tabular}{cccc}
\hline Entry & Temperature $/{ }^{\circ} \mathrm{C}$ & Yield $^{b} / \%$ & $e e^{c} / \%$ \\
\hline 1 & 15 & 53 & 20 \\
2 & 20 & 81 & 20 \\
3 & 25 & 87 & 17 \\
4 & 30 & 89 & 14 \\
5 & 40 & 94 & 9 \\
6 & 50 & 97 & 14 \\
7 & 60 & 88 & 12 \\
\hline
\end{tabular}

质应条件: 1 (0.5 mmol), 2a (1.5 mmol, 3 equiv.), 虹蚂粗提物 $(100 \mathrm{mg})$, 二 甲亚砜 $(0.90 \mathrm{~mL})$ 和去离子水 $(0.10 \mathrm{~mL})$, 反应 $120 \mathrm{~h} ;{ }^{b}$ 收率是通过硅胶色谱 柱分离得到; ${ }^{c} e e$ 是通过手性高效液相色谱(Chiralpak AD-H 手性柱)分析得 到.

为了探究蚯蚂粗提物在 Michael 反应中的作用, 我 们开展了一系列对照实验. 首先, 在无催化剂的条件下, 空白实验只检测到痕量的产物生成(表 6, Entry 1). 在虹 蚂粗提物催化下得到的 $97 \%$ 收率和 $14 \% e e$ (表 6 , Entry 2), 表明蝶蚓粗提物确实催化了该 Michael 反应. 由于 虾蚓消化道内存在大量丝氨酸蛋白酶, 因此使用丝氨酸 蛋白酶的抑制剂苯甲基磺酰氟(PMSF) ${ }^{[19]}$ 预处理虾蚓粗 提物, 经 PMSF 处理过的蝶蚓粗提物用于催化模型反应, 得到 63\%收率和 3\% ee(表 6, Entry 3). PMSF 本身对该 Michael 反应也有一定的催化作用，用等量的 PMSF 催 化得到 43\%收率(表 6, Entry 4). 除去 PMSF 的影响, 可 以推测 PMSF 抑制的虾蚂粗提物参与的催化只有 $20 \%$ 的 收率，相比较于未经抑制的蝶蚓粗提物催化得到的 $97 \%$ 
收率, PMSF 显著地抑制了蚯蚓粗提物的活性, 使收率 下降了 $77 \%$. 上述实验表明，蝶蚓粗提物确实催化了该 Michael 反应. 最后, 对蚯蚂粗提物进行了蛋白酶活力 测定(测定蛋白酶的水解活性). 采用 Sigma 公司提供的 酪蛋白为底物的蛋白酶活力测定实验方法, 测得蝶蚓粗 提物的蛋白酶活力为 $0.1 \mathrm{U} / \mathrm{mg}$ (1961 年世界生物化学协 会酶学委员会用国际单位 $U$ 来定义酶的活性, 定义为在 一定的标准条件下, $1 \mathrm{U}$ 即每分钟催化 $1 \mathrm{~mol}$ 底物的酶 量 $^{[20]}$ ). 酶活实验表明, 蚯蚓粗提物中确实含有蛋白酶.

表 6 对照实验 ${ }^{a}$

Table 6 Control experiments

\begin{tabular}{|c|c|c|c|}
\hline Entry & 催化剂 & Yield $^{b} / \%$ & $e e^{c} / \%$ \\
\hline 1 & None & Trace & - \\
\hline 2 & 虾蚓粗提物(100 mg) & 97 & 14 \\
\hline 3 & $\begin{array}{l}\text { PMSF 处理过的蚳蚓粗 } \\
\text { 提物 }(100 \mathrm{mg})^{d}\end{array}$ & 63 & 3 \\
\hline 4 & PMSF (106 mg) & 43 & - \\
\hline
\end{tabular}

${ }^{a}$ 反应条件: $1(0.5 \mathrm{mmol}), \mathbf{2 a}(1.5 \mathrm{mmol}, 3$ equiv.), 催化剂, 二甲亚砜 $(0.90$ $\mathrm{mL})$ 和水 $(0.10 \mathrm{~mL})$ 在 $50{ }^{\circ} \mathrm{C}$ 下反应 $120 \mathrm{~h} ;{ }^{b}$ 收率是通过硅胶色谱柱分离得 到; ${ }^{c} e e$ 是通过手性高效液相色谱(Chiralpak AD-H 手性柱)分析得到; ${ }^{d}$ 虹 蚂粗提物 $(100 \mathrm{mg})$ 和 PMSF $(106 \mathrm{mg})$ 加入到 THF $(1 \mathrm{~mL})$ 中, 在 $30{ }^{\circ} \mathrm{C}$ 下摚 拌反应 $4 \mathrm{~h}$ 后减压蒸馏除去 $\mathrm{THF}$, 再用于催化反应

接着，探究了反应时间对蚯蚓粗提物催化的 Michael 反应的影响. 在反应时间为 $24 、 36 、 48 \mathrm{~h}$ 下, 模 型反应分别得到 $71 \%$ 、83\%、98\%的收率，随着时间的 延长, $e e$ 变化不大 $(13 \% \sim 15 \%)$. 这一实验表明, 在优化 的条件下模型反应在 $48 \mathrm{~h}$ 的时候已经进行得很充分.

在此基础上，我们对蚳蚓粗提物催化的 Michael 反 应进行了底物的扩展. 考察了不同取代的芐叉丙酮作为 底物时的情况. 发现, 无论苯环上取代的是供电子还是 吸电子基团, 都能得到满意的收率. 其中, 无取代的芐 叉丙酮得到最高收率 98\%(表 7, Entry 1). 3-甲氧基苄叉 丙酮得到 91\%的收率(表 7, Entry 3)，但是 2-和 4-甲氧基 芐叉丙酮得到收率较低(表 7, Entries 2 和 4), 可能是由 于甲氧基推电子的共轭效应所致. 2-和 3-氯取代苄叉丙 酮得到收率较 4-氯取代芐叉丙酮高(表 7, Entries 5 7). 氟取代的茮叉丙酮也能得到较好的收率(表 7, Entries $8 \sim 10)$. 该方法还适用于菜取代的烯酮，得到高达 $99 \%$ 的收率和 $16 \%$ 的 $e e$ (表 7, Entry 11). 说明蚯蚂粗提物催 化 Michael 反应的普适性较好. 在所考察的底物中, 对 映选择性最高达到 $20 \% e e$ (表 7, Entry 8). 所有产物均为 已知化合物, 物理性质和表征数据与参考文献一 致 ${ }^{[4,13,21]}$.

\section{2 结论}

通过蚳蚓粗提物催化的 Michael 反应成功合成了抗
表 7 虾蚓粗提物催化的 Michael 反应的底物扩展 ${ }^{a}$

Table 7 Substrate scope of the crude earthwarm extract catalyzed-Michael reaction ${ }^{a}$<smiles>CC(=O)CC([Al])C1=C(O)C2=CC3=CC=C(OC(=O)C2=C1)C(=O)[Te]C=CC(=O)CO3</smiles>

123

\begin{tabular}{clcccc}
\hline Entry & \multicolumn{1}{c}{$\mathrm{Ar}$} & 产物 & $t / \mathrm{h}$ & $\mathrm{Yield}^{b} / \%$ & $e e^{c} / \%$ \\
\hline 1 & $\mathrm{Ph}$ & $\mathbf{3 a}$ & 48 & 98 & 13 \\
2 & $2-\mathrm{MeOC}_{6} \mathrm{H}_{4}$ & 3b & 47 & 57 & 0 \\
3 & $3-\mathrm{MeOC}_{6} \mathrm{H}_{4}$ & $\mathbf{3 c}$ & 50 & 91 & 12 \\
4 & $4-\mathrm{MeOC}_{6} \mathrm{H}_{4}$ & 3d & 52 & 73 & 10 \\
5 & $2-\mathrm{ClC}_{6} \mathrm{H}_{4}$ & $\mathbf{3 e}$ & 55 & 88 & 11 \\
6 & $3-\mathrm{ClC}_{6} \mathrm{H}_{4}$ & $\mathbf{3 f}$ & 52 & 89 & 15 \\
7 & $4-\mathrm{ClC}_{6} \mathrm{H}_{4}$ & $\mathbf{3 g}$ & 33 & 73 & 15 \\
8 & $2-\mathrm{FC}_{6} \mathrm{H}_{4}$ & $\mathbf{3 h}$ & 60 & 81 & 0 \\
9 & $3-\mathrm{FC}_{6} \mathrm{H}_{4}$ & $\mathbf{3 i}$ & 60 & 89 & 20 \\
10 & $4-\mathrm{FC}_{6} \mathrm{H}_{4}$ & $\mathbf{3 j}$ & 37 & 86 & 10 \\
11 & $2-\mathrm{Naphthyl}_{4}$ & $\mathbf{3 k}$ & 47 & 99 & 16 \\
\hline
\end{tabular}

${ }^{a}$ 反应条件: 1 ( $\left.0.5 \mathrm{mmol}\right), 2$ (1.5 mmol, 3 equiv.), 虾蚂粗提物 $(100 \mathrm{mg})$, 二 甲亚砜 $(0.90 \mathrm{~mL})$, 水 $(0.10 \mathrm{~mL})$, 反应温度 $50{ }^{\circ} \mathrm{C} ;{ }^{b}$ 收率是通过硅胶色谱柱 分离得到; ${ }^{c} e e$ 是通过手性高效液相色谱(Chiralpak AD-H 手性柱)分析得 到.

凝血药华法林及其一系列衍生物，并获得良好的收率 (最高可达 99\%). 虽然对映选择性不太理想，但该方法 以虾蚓粗提物这一价廉易得、制备简单的天然物质作为 绿色环保可再生的生物催化剂, 为华法林的合成提供了 一种高效、简洁的方法.

\section{3 实验部分}

\section{1 仪器与试剂}

核磁共振波谱分析仪 Bruker-AM 600(600 MHz); 显微熔点仪 WPX-4(上海易测仪器设备有限公司). 蝶蚓 粗提物，由我们课题组制备 ${ }^{[14]}$. 取代苄叉丙酮 $\mathbf{2 b}, \mathbf{2 c}$, 2e, 2f, 2h, 2i, 2k 制备方法参见文献[22]. 2d, 2g, 2j 制备 方法参照文献[23]. 所有试剂均为分析纯市售品而未经 进一步纯化.

\section{2 实验方法}

在圆底烧瓶中依次加入 4-羟基香豆素(1) (0.5 $\mathrm{mmol})$ 、苄叉丙酮或其衍生物 $2(1.5 \mathrm{mmol})$ 、蝶蚂粗提物 $(100 \mathrm{mg})$ 、二甲亚砜 $(0.90 \mathrm{~mL})$ 和水 $(0.10 \mathrm{~mL})$, 混合物在 $50{ }^{\circ} \mathrm{C}$ 下搅拌反应，通过薄层色谱(TLC)监测. 待反应结 束, 在反应瓶中加入乙酸乙酯 $(4 \mathrm{~mL})$, 通过抽滤除去蚯 蚂粗提物, 再用乙酸乙酷洗涤滤渣, 滤液用水洗涤, 有 机相经无水硫酸钠干燥, 真空抽滤去除无水硫酸钠, 再 通过减压蒸馏除去溶剂, 然后将粗产物通过柱层析纯化 分离得到纯的目标产物. 


\section{3 实验数据}

所有产物均为已知化合物, 物理性质和表征数据与 参考文献一致 ${ }^{[4,7,13,21,24]}$.

4-羟基-3-(3-氧-1-苯丁基)-苯并吡喃-2-酮(3a): 得 $151 \mathrm{mg}$, 产率 98\%. 白色粉末, m.p. 163 164 ${ }^{\circ} \mathrm{C}$ (文献 值 $\left.{ }^{[7]} 169 \sim 171{ }^{\circ} \mathrm{C}\right) ;{ }^{1} \mathrm{H}$ NMR $\left(600 \mathrm{MHz}, \mathrm{CDCl}_{3}\right) \delta: 9.49$ (br. s, $0.12 \mathrm{H}), 7.96$ (d, $J=7.9 \mathrm{~Hz}, 0.18 \mathrm{H}), 7.92$ (d, $J=7.9$ $\mathrm{Hz}, 0.50 \mathrm{H}), 7.83(\mathrm{~d}, J=7.5 \mathrm{~Hz}, 0.59 \mathrm{H}), 7.58$ (t, $J=7.8 \mathrm{~Hz}$, $0.50 \mathrm{H}), 7.50(\mathrm{~d}, J=7.9 \mathrm{~Hz}, 0.68 \mathrm{H}), 7.35 \sim 7.23(\mathrm{~m}$, $7.58 \mathrm{H}$ ), 4.74 (d, $J=8.6 \mathrm{~Hz}, 0.18 \mathrm{H}), 4.29$ (dd, $J=6.4,3.1$ $\mathrm{Hz}, 0.50 \mathrm{H}), 4.19(\mathrm{dd}, J=11.2,6.9 \mathrm{~Hz}, 0.60 \mathrm{H}), 3.87$ (dd, $J=19.4,10.0 \mathrm{~Hz}, 0.19 \mathrm{H}), 3.32$ (s, $0.13 \mathrm{H}), 2.58 \sim 2.40$ (m, $1.75 \mathrm{H}), 2.30(\mathrm{~s}, 0.50 \mathrm{H}), 2.03(\mathrm{dd}, J=24.9,13.0 \mathrm{~Hz}$, $0.71 \mathrm{H}), 1.72(\mathrm{~s}, 1.68 \mathrm{H}), 1.69(\mathrm{~s}, 1.38 \mathrm{H}) ;{ }^{13} \mathrm{C}$ NMR $(151$ $\left.\mathrm{MHz}, \mathrm{CDCl}_{3}\right) \delta: 162.1,161.0,159.7,158.8,153.0,153.0$, $143.2,141.6,129.2,128.6,127.1,127.0,123.8,123.6$, $116.6,116.5,116.0,104.2,101.3,100.6,99.1,45.2,42.6$, 40.2, 35.4, 35.0, 34.4, 30.0, 28.1, 27.6.

4-羟基-3-[1-(2-甲氧苯基)-3-氧代丁基]-苯并吡喃2-酮(3b): 得 $96 \mathrm{mg}$, 产率 57\%. 白色粉末, m.p. 181 $183{ }^{\circ} \mathrm{C}$ (文献值 ${ }^{[7]} 198 \sim 199{ }^{\circ} \mathrm{C}$ ); ${ }^{1} \mathrm{H}$ NMR $(600 \mathrm{MHz}$, $\left.\mathrm{CDCl}_{3}\right) \delta: 9.15$ (s, 0.38H), $7.93(\mathrm{t}, J=9.3 \mathrm{~Hz}, 1.15 \mathrm{H}), 7.84$ (d, $J=7.8 \mathrm{~Hz}, 0.36 \mathrm{H}), 7.72(\mathrm{~d}, J=7.6 \mathrm{~Hz}, 0.39 \mathrm{H}), 7.58$ (t, $J=7.7 \mathrm{~Hz}, 0.79 \mathrm{H}), 7.50(\mathrm{dt}, J=22.0,7.7 \mathrm{~Hz}, 0.82 \mathrm{H})$, $7.40 \sim 7.19(\mathrm{~m}, 5.37 \mathrm{H}), 7.11 \sim 6.84(\mathrm{~m}, 4.36 \mathrm{H}), 4.99(\mathrm{dd}$, $J=8.2,5.5 \mathrm{~Hz}, 0.44 \mathrm{H}), 4.60(\mathrm{~s}, 0.34 \mathrm{H}), 4.56(\mathrm{~d}, J=5.9$ $\mathrm{Hz}, 0.94 \mathrm{H}), 3.97$ (s, 1.18H), 3.93 (s, 2.32H), $3.86(\mathrm{~s}$, $1.29 \mathrm{H}), 3.50$ (s, $0.77 \mathrm{H}), 3.27$ (s, $0.37 \mathrm{H}), 2.68$ (dd, $J=14.5$, $1.7 \mathrm{~Hz}, 0.80 \mathrm{H}), 2.51$ (dd, $J=13.8,6.9 \mathrm{~Hz}, 0.41 \mathrm{H}), 2.32$ (dd, $J=14.5,7.1 \mathrm{~Hz}, 0.80 \mathrm{H}), 2.21$ (s, $1.18 \mathrm{H}), 2.06$ (d, $J=$ $6.6 \mathrm{~Hz}, 0.52 \mathrm{H}), 1.72(\mathrm{~s}, 0.99 \mathrm{H}), 1.69(\mathrm{~s}, 2.27 \mathrm{H}) ;{ }^{13} \mathrm{C} \mathrm{NMR}$ $\left(151 \mathrm{MHz}, \mathrm{CDCl}_{3}\right) \delta: 162.2,161.9,160.9,159.7,157.2$, $157.0,155.4,153.0,152.9,152.7,131.8,131.5,131.3$, $129.0,128.7,128.0,127.5,126.4,123.8,123.8,123.6$, $123.5,123.0,122.6,121.8,121.0,120.8,116.6,116.5$, $116.4,116.1,115.7,111.4,111.0,110.9,106.3,101.3$, $100.7,99.2,56.2,55.6,55.4,45.4,37.1,30.2,29.3,28.2$, 28.1.

4-羟基-3-[1-(3-甲氧苯基)-3-氧代丁基]-苯并吡喃2-酮 (3c) ${ }^{[24]}$ : 得 $154 \mathrm{mg}$, 产率 $91 \%$. 白色粉末, m.p. $100 \sim 102{ }^{\circ} \mathrm{C} ;{ }^{1} \mathrm{H}$ NMR $\left(600 \mathrm{MHz}, \mathrm{CDCl}_{3}\right) \delta: 9.42(\mathrm{~s}$, $0.08 \mathrm{H}), 7.92(\mathrm{~d}, J=7.8 \mathrm{~Hz}, 0.36 \mathrm{H}), 7.84(\mathrm{~d}, J=7.8 \mathrm{~Hz}$, $0.38 \mathrm{H}), 7.61 \sim 7.50(\mathrm{~m}, 0.89 \mathrm{H}), 7.40 \sim 7.24(\mathrm{~m}, 2.56 \mathrm{H})$, $7.23(\mathrm{~d}, J=7.1 \mathrm{~Hz}, 0.39 \mathrm{H}), 6.84$ (ddd, $J=38.3,17.8,7.8$ $\mathrm{Hz}, 2.60 \mathrm{H}), 4.71(\mathrm{~d}, J=8.0 \mathrm{~Hz}, 0.12 \mathrm{H}), 4.29$ (d, $J=4.2$
$\mathrm{Hz}, 0.39 \mathrm{H}), 4.16(\mathrm{dd}, J=11.2,6.9 \mathrm{~Hz}, 0.41 \mathrm{H}), 3.84(\mathrm{dd}$, $J=19.4,9.9 \mathrm{~Hz}, 0.18 \mathrm{H}), 3.79$ (s, 2.47H), 3.49 3.23 (m, $0.89 \mathrm{H}$ ), 2.57 (dd, $J=14.2,2.6 \mathrm{~Hz}, 0.39 \mathrm{H}), 2.50$ (dd, $J=$ 14.0, $6.9 \mathrm{~Hz}, 0.40 \mathrm{H}), 2.43(\mathrm{dd}, J=14.2,7.0 \mathrm{~Hz}, 0.41 \mathrm{H})$, $2.31(\mathrm{~s}, 0.30 \mathrm{H}), 2.08 \sim 1.98(\mathrm{~m}, 0.46 \mathrm{H}), 1.74(\mathrm{~s}, 1.13 \mathrm{H})$, $1.70(\mathrm{~s}, 1.45 \mathrm{H}) ;{ }^{13} \mathrm{C}$ NMR $\left(151 \mathrm{MHz}, \mathrm{CDCl}_{3}\right) \delta: 162.0$, $161.1,160.4,159.8,159.6,158.6,153.1,153.0,152.8$, $144.9,143.2,132.0,131.5,130.4,129.6,129.2,123.9$, $123.8,123.6,123.1,122.7,120.3,116.7,116.5,116.2$, $115.9,115.6,114.4,113.4,111.6,104.2,101.1,100.5,99.0$, $55.2,55.2,45.3,42.5,40.0,35.4,35.0,34.1,30.0,28.2$, 27.8 .

4-羟基-3-[1-(4-甲氧苯基)-3-氧代丁基]-苯并吡喃2-酮(3d): 得 $123 \mathrm{mg}$, 产率 73\%. 白色粉末. m.p. 53 $55{ }^{\circ} \mathrm{C}$ (文献值 ${ }^{[7]} 164 \sim 166{ }^{\circ} \mathrm{C}$ ); ${ }^{1} \mathrm{H}$ NMR $(600 \mathrm{MHz}$, $\left.\mathrm{CDCl}_{3}\right) \delta: 9.45$ (s, 0.15H), $7.92(\mathrm{dd}, J=17.2,7.9 \mathrm{~Hz}$, $0.60 \mathrm{H}), 7.81(\mathrm{~d}, J=8.0 \mathrm{~Hz}, 0.66 \mathrm{H}), 7.57(\mathrm{dd}, J=11.3,4.3$ $\mathrm{Hz}, 0.49 \mathrm{H}), 7.48(\mathrm{dd}, J=12.2,4.6 \mathrm{~Hz}, 0.74 \mathrm{H}), 7.36 \sim 7.09$ $(\mathrm{m}, 5.74 \mathrm{H}), 6.85(\mathrm{dd}, J=12.0,8.8 \mathrm{~Hz}, 2.42 \mathrm{H}), 4.69(\mathrm{dd}$, $J=9.7,2.4 \mathrm{~Hz}, 0.16 \mathrm{H}), 4.23$ (dd, $J=6.4,3.3 \mathrm{~Hz}, 0.50 \mathrm{H}$ ), 4.14 (dd, $J=11.2,6.8 \mathrm{~Hz}, 0.79 \mathrm{H}), 3.87 \sim 3.80(\mathrm{~m}, 0.32 \mathrm{H})$, $3.78(\mathrm{~d}, J=5.1 \mathrm{~Hz}, 3.55 \mathrm{H}), 3.61(\mathrm{~s}, 0.55 \mathrm{H}), 2.53 \sim 2.34$ (m, 1.75H), $2.28(\mathrm{~s}, 0.47 \mathrm{H}), 2.00(\mathrm{dd}, J=13.8,11.4 \mathrm{~Hz}$, $0.65 \mathrm{H}), 1.70$ (s, $1.49 \mathrm{H}), 1.68(\mathrm{~s}, 1.50 \mathrm{H}) ;{ }^{13} \mathrm{C}$ NMR $(151$ $\left.\mathrm{MHz}, \mathrm{CDCl}_{3}\right) \delta: 162.2,161.4,159.6,158.8,158.7,158.2$, $152.9,133.3,131.4,128.1,123.8,123.6,123.1,116.6$, 116.4, 114.6, 113.7, 104.4, 101.6, 100.7, 99.2, 55.3, 55.2, $34.4,33.6,28.0,27.6$.

4-羟基-3-[1-(2-氯苯基)-3-氧代丁基]-苯并吡喃-2酮(3e $)^{[4,13,21]}$ : 得 $150 \mathrm{mg}$ 白色粉末, 产率 88\%. m.p. 168 $170{ }^{\circ} \mathrm{C} ;{ }^{1} \mathrm{H}$ NMR $(600 \mathrm{MHz}, \mathrm{CDCl} 3) \delta: 9.59$ (s, 0.17H), 7.99 (d, $J=7.9 \mathrm{~Hz}, 0.17 \mathrm{H}), 7.90(\mathrm{~d}, J=7.8 \mathrm{~Hz}, 0.57 \mathrm{H})$, $7.83(\mathrm{~d}, J=7.8 \mathrm{~Hz}, 0.69 \mathrm{H}), 7.71(\mathrm{~d}, J=7.8 \mathrm{~Hz}, 0.18 \mathrm{H})$, 7.53 (ddd, $J=27.6,17.7,10.1 \mathrm{~Hz}, 1.53 \mathrm{H}), 7.42$ (d, $J=7.8$ $\mathrm{Hz}, 0.63 \mathrm{H}), 7.40 \sim 7.26(\mathrm{~m}, 3.87 \mathrm{H}), 7.15$ (td, $J=15.1,7.6$ $\mathrm{Hz}, 3.96 \mathrm{H}), 4.85$ (d, $J=10.8 \mathrm{~Hz}, 0.20 \mathrm{H}), 4.59-4.53$ (m, $0.59 \mathrm{H}), 4.14$ (q, $J=7.1 \mathrm{~Hz}, 0.66 \mathrm{H}), 3.99$ (dd, $J=19.0,11.0$ $\mathrm{Hz}, 0.81 \mathrm{H}), 3.48$ (s, $0.55 \mathrm{H}), 3.20$ (d, $J=19.1 \mathrm{~Hz}, 0.18 \mathrm{H})$, $2.57(\mathrm{dd}, J=14.5,3.3 \mathrm{~Hz}, 1.33 \mathrm{H}), 2.38(\mathrm{dd}, J=14.4,7.5$ $\mathrm{Hz}, 0.64 \mathrm{H}), 2.30(\mathrm{~s}, 0.47 \mathrm{H}), 2.06(\mathrm{~s}, 1.00 \mathrm{H}), 1.75(\mathrm{~s}$, $2.53 \mathrm{H}), 1.71$ (s, $1.64 \mathrm{H}) ;{ }^{13} \mathrm{C}$ NMR $\left(151 \mathrm{MHz}, \mathrm{CDCl}_{3}\right) \delta$ : $161.7,161.0,153.0,153.0,139.0,133.8,132.0,131.6$, $130.3,129.2,128.2,128.1,127.7,127.1,126.8,123.9$, $123.7,122.9,122.7,116.7,116.6,116.1,115.9,115.6$, 101.3, 100.4, 99.1, 45.9, 37.2, 32.7, 32.5, 30.0, 28.2, 28.0. 
4-羟基-3-[1-(3-氯苯基)-3-氧代丁基]-苯并吡喃-2酮(3f $)^{[13,21]}$ : 得 $152 \mathrm{mg}$, 产率 $89 \%$. 白色粉末 m.p. 162 $164{ }^{\circ} \mathrm{C} ;{ }^{1} \mathrm{H}$ NMR $\left(600 \mathrm{MHz}, \mathrm{CDCl}_{3}\right) \delta$ : 9.56 (s, $\left.0.19 \mathrm{H}\right)$, $7.98(\mathrm{~d}, J=7.6 \mathrm{~Hz}, 0.19 \mathrm{H}), 7.90(\mathrm{~d}, J=7.8 \mathrm{~Hz}, 0.47 \mathrm{H})$, $7.84(\mathrm{~d}, J=7.2 \mathrm{~Hz}, 0.71 \mathrm{H}), 7.56(\mathrm{dt}, J=14.5,7.9 \mathrm{~Hz}$, $1.46 \mathrm{H}), 7.39 \sim 7.13(\mathrm{~m}, 8.92 \mathrm{H}), 4.68(\mathrm{~d}, J=9.9 \mathrm{~Hz}$, $0.21 \mathrm{H}), 4.22(\mathrm{dd}, J=6.8,3.5 \mathrm{~Hz}, 0.53 \mathrm{H}), 4.19 \sim 4.09(\mathrm{~m}$, $0.98 \mathrm{H}), 3.86(\mathrm{dd}, J=19.3,10.4 \mathrm{~Hz}, 0.25 \mathrm{H}), 3.18$ (s, $0.47 \mathrm{H}), 2.53 \sim 2.37(\mathrm{~m}, 1.85 \mathrm{H}), 2.32(\mathrm{~s}, 0.60 \mathrm{H}), 1.98(\mathrm{t}$, $J=12.8 \mathrm{~Hz}, 0.80 \mathrm{H}), 1.76$ (s, 2.14H), $1.72(\mathrm{~s}, 1.40 \mathrm{H}) ;{ }^{13} \mathrm{C}$ NMR $\left(151 \mathrm{MHz}, \mathrm{CDCl}_{3}\right) \delta: 161.9,161.1,159.7,159.0$, 153.0, 153.0, 145.4, 144.3, 134.7, 134.4, 132.1, 132.0, $131.7,129.9,129.8,127.6,127.1,127.1,126.8,125.5$, $125.4,123.9,123.7,123.0,122.8116 .7,116.6,115.8$, $115.5,103.6,101.1,100.2,98.9,42.3,40.0,35.2,34.6$, 28.2, 27.7 .

4-差基-3-[1-(4-氯苯基)-3-氧代丁基]-苯并吡喃-2酮(3g): 得 $125 \mathrm{mg}$, 产率 73\%. 白色粉末, m.p. 166 $168{ }^{\circ} \mathrm{C}$ (文献值 ${ }^{[7]} 174 \sim 176{ }^{\circ} \mathrm{C}$ ); ${ }^{1} \mathrm{H}$ NMR $(600 \mathrm{MHz}$, $\left.\mathrm{CDCl}_{3}\right) \delta: 9.59(\mathrm{~s}, 0.10 \mathrm{H}), 7.95(\mathrm{~d}, J=7.9 \mathrm{~Hz}, 0.13 \mathrm{H}), 7.88$ (d, $J=7.3 \mathrm{~Hz}, 0.30 \mathrm{H}), 7.81(\mathrm{~d}, J=7.3 \mathrm{~Hz}, 0.47 \mathrm{H}), 7.52$ (ddd, $J=19.7,13.5,7.3 \mathrm{~Hz}, 0.96 \mathrm{H}), 7.35 \sim 7.13(\mathrm{~m}$, $6.21 \mathrm{H}), 4.68(\mathrm{~d}, J=9.8 \mathrm{~Hz}, 0.11 \mathrm{H}), 4.16(\mathrm{dd}, J=11.3,6.8$ $\mathrm{Hz}, 0.81 \mathrm{H}), 3.82$ (dd, $J=19.2,10.0 \mathrm{~Hz}, 0.20 \mathrm{H}), 3.29$ (d, $J=19.1 \mathrm{~Hz}, 0.16 \mathrm{H}), 2.45(\mathrm{dd}, J=13.8,7.1 \mathrm{~Hz}, 0.84 \mathrm{H})$, 2.37 (dd, $J=14.0,7.1 \mathrm{~Hz}, 0.35 \mathrm{H}), 2.29$ (s, $0.33 \mathrm{H}), 1.95$ (t, $J=12.7 \mathrm{~Hz}, 0.56 \mathrm{H}), 1.72(\mathrm{~s}, 1.47 \mathrm{H}), 1.69(\mathrm{~s}, 0.98 \mathrm{H}) ;{ }^{13} \mathrm{C}$ NMR $\left(151 \mathrm{MHz}, \mathrm{CDCl}_{3}\right) \delta: 162.1,161.4,159.8,159.1$, $153.0,152.9,152.8,141.8,140.7,138.5,132.5,132.4$, $132.1,132.1,131.9,131.7,129.4,128.8,128.7,128.7$, $128.5,128.2,124.0,123.9,123.7,123.0,122.8,116.6$, $116.5,116.2,115.8,115.6,107.5,103.7,101.3,100.4$, $99.1,45.2,42.4,40.1,34.9,34.4,30.0,28.0,27.6$.

4-差基-3-[1-(2-氟苯基)-3-氧代丁基]-苯并吡喃-2酮(3h): 得 $132 \mathrm{mg}$, 白色粉末, 产率 81\%. m.p. 166 $168{ }^{\circ} \mathrm{C} ;{ }^{1} \mathrm{H}$ NMR (600 MHz, DMSO) $\delta: 7.93 \sim 7.59(\mathrm{~m}$, $2.09 \mathrm{H}), 7.41 \sim 7.01(\mathrm{~m}, 6.12 \mathrm{H}), 4.28(\mathrm{~d}, J=43.7 \mathrm{~Hz}$, $0.96 \mathrm{H}), 3.36(\mathrm{~s}, 0.91 \mathrm{H}), 2.60 \sim 2.00(\mathrm{~m}, 1.86 \mathrm{H}), 1.66(\mathrm{~d}$, $J=52.2 \mathrm{~Hz}, 3.00 \mathrm{H}) ;{ }^{13} \mathrm{C}$ NMR $(151 \mathrm{MHz}, \mathrm{DMSO}) \delta$ : $161.5,159.9\left(\mathrm{~d},{ }^{1} J_{\mathrm{C}-\mathrm{F}}=241.8 \mathrm{~Hz}\right), 152.8,132.4,130.6$, $130.2,129.3,128.3,124.8,124.4,123.2,116.6,115.5$, 103.1, 101.4, 100.0, 30.0, 29.0, 27.7, 26.8 .

4-羟基-3-[1-(3-氟苯基)-3-氧代丁基]-苯并吡喃-2酮(3i) ${ }^{[4]}$ : 得 $146 \mathrm{mg}$, 产率 $89 \%$. 白色粉末, m.p. 162 $164{ }^{\circ} \mathrm{C} ;{ }^{1} \mathrm{H}$ NMR $\left(600 \mathrm{MHz}, \mathrm{CDCl}_{3}\right) \delta$ : 7.98 (d, $J=7.4$
$\mathrm{Hz}, 0.14 \mathrm{H}), 7.90$ (d, $J=7.8 \mathrm{~Hz}, 0.37 \mathrm{H}), 7.84$ (d, $J=7.8$ $\mathrm{Hz}, 0.50 \mathrm{H}), 7.56$ (dt, $J=36.0,7.8 \mathrm{~Hz}, 1.07 \mathrm{H}), 7.31$ (tdd, $J=24.7,16.8,7.6 \mathrm{~Hz}, 3.53 \mathrm{H}), 7.10 \sim 6.89(\mathrm{~m}, 3.11 \mathrm{H}), 4.71$ (d, $J=10.0 \mathrm{~Hz}, 0.14 \mathrm{H}), 4.26(\mathrm{dd}, J=6.8,3.3 \mathrm{~Hz}, 0.37 \mathrm{H})$, $4.22 \sim 4.12(\mathrm{~m}, 0.60 \mathrm{H}), 3.86(\mathrm{dd}, J=19.0,9.9 \mathrm{~Hz}, 0.16 \mathrm{H})$, $3.50(\mathrm{~s}, 0.55 \mathrm{H}), 3.32(\mathrm{~d}, J=19.4 \mathrm{~Hz}, 0.19 \mathrm{H}), 3.23$ (s, $0.39 \mathrm{H}$ ), 2.50 (ddd, $J=13.6,8.2,5.4 \mathrm{~Hz}, 0.92 \mathrm{H}), 2.43$ (dd, $J=14.2,7.2 \mathrm{~Hz}, 0.44 \mathrm{H}), 2.32(\mathrm{~s}, 0.32 \mathrm{H}), 2.02 \sim 1.95(\mathrm{~m}$, $0.57 \mathrm{H}), 1.76(\mathrm{~s}, 1.60 \mathrm{H}), 1.72(\mathrm{~s}, 1.28 \mathrm{H}) ;{ }^{13} \mathrm{C}$ NMR $(151$ $\left.\mathrm{MHz}, \mathrm{CDCl}_{3}\right) \delta: 162.0,161.2,159.0,153.0,153.0,132.1$, $131.7,130.3,130.3,130.0,130.0,124.0,123.7,123.0$, $122.8,122.7,116.7,116.6,116.2,115.8,114.5,114.3$, $113.9,113.9,113.8,113.5,113.4,103.7,100.3,99.0,77.2$, $77.0,76.8,50.8,42.3,40.0,35.2,34.5,30.0,28.2,27.7$.

4-羟基-3-[1-(4-氟苯基)-3-氧代丁基]-苯并吡喃-2酮 $(3 \mathbf{j})^{[4,13]}$ : 得 $140 \mathrm{mg}$, 产率 $86 \%$. 白色粉末, m.p. 171 $172{ }^{\circ} \mathrm{C} ;{ }^{1} \mathrm{H}$ NMR (600 MHz, $\left.\mathrm{CDCl}_{3}\right) \delta$ : 9.54 (s, 0.13H), $7.97(\mathrm{~d}, J=7.9 \mathrm{~Hz}, 0.14 \mathrm{H}), 7.90(\mathrm{~d}, J=7.8 \mathrm{~Hz}, 0.33 \mathrm{H})$, $7.83(\mathrm{~d}, J=7.6 \mathrm{~Hz}, 0.50 \mathrm{H}), 7.60 \sim 7.49(\mathrm{~m}, 1.01 \mathrm{H}), 7.37 \sim$ $7.17(\mathrm{~m}, 4.41 \mathrm{H}), 7.03 \sim 6.95(\mathrm{~m}, 1.95 \mathrm{H}), 4.69(\mathrm{~d}, J=9.8$ $\mathrm{Hz}, 0.13 \mathrm{H}), 4.23(\mathrm{~s}, 0.33 \mathrm{H}), 4.20 \sim 4.12(\mathrm{~m}, 0.74 \mathrm{H}), 3.87$ (dd, $J=19.4,10.3 \mathrm{~Hz}, 0.19 \mathrm{H}), 3.59$ (s, 0.49H), 3.31 (s, $0.09 \mathrm{H}$ ), 3.27 (d, $J=6.3 \mathrm{~Hz}, 0.40 \mathrm{H}$ ), 2.47 (dd, $J=13.9,6.6$ $\mathrm{Hz}, 0.82 \mathrm{H}), 2.41(\mathrm{dd}, \quad J=14.0,6.9 \mathrm{~Hz}, 0.39 \mathrm{H}), 2.31(\mathrm{~s}$, $0.38 \mathrm{H}), 2.02 \sim 1.95(\mathrm{~m}, 0.55 \mathrm{H}), 1.75(\mathrm{~s}, 1.56 \mathrm{H}), 1.71(\mathrm{~s}$, $1.27 \mathrm{H}) ;{ }^{13} \mathrm{C}$ NMR $\left(151 \mathrm{MHz}, \mathrm{CDCl}_{3}\right) \delta: 161.2,160.7(\mathrm{~d}$, $\left.{ }^{1} J_{\mathrm{C}-\mathrm{F}}=73.4 \mathrm{~Hz}\right), 159.6,158.8\left(\mathrm{~d},{ }^{1} J_{\mathrm{C}-\mathrm{F}}=118.0 \mathrm{~Hz}\right), 153.0$, $153.0,138.8,132.0,131.8,131.6,129.6,129.6,128.8$, $128.7,128.6,128.5,123.9,123.9,123.7,123.0,122.7$, $116.7,116.5,116.2,115.8,115.8,115.6,115.6,115.5$, $115.3,115.0,114.8,104.1,101.5,100.4,99.0,45.5,42.6$, 40.1, 34.7, 34.4, 34.0, 30.0, 28.2, 27.7.

4-羟基-3-(2-菜-3-氧代丁基)-苯并吡喃-2-酮(3k $)^{[13]}$ : 得 $177 \mathrm{mg}$, 产率 99\%. 白色粉末, m.p. $117 \sim 119{ }^{\circ} \mathrm{C} ;{ }^{1} \mathrm{H}$ NMR $\left(600 \mathrm{MHz}, \mathrm{CDCl}_{3}\right) \delta: 7.95 \sim 7.64(\mathrm{~m}, 4.71 \mathrm{H})$, $7.57 \sim 7.17(\mathrm{~m}, 5.96 \mathrm{H}), 4.41(\mathrm{~s}, 0.95 \mathrm{H}), 2.78 \sim 2.24(\mathrm{~m}$, $1.84 \mathrm{H}), 1.70$ (s, $3.00 \mathrm{H}) ;{ }^{13} \mathrm{C}$ NMR $\left(151 \mathrm{MHz}, \mathrm{CDCl}_{3}\right) \delta$ : 153.0, 133.6, 132.5, 131.8, 127.8, 127.6, 126.1, 125.6, $123.8,123.1,116.6,26.9$.

辅助材料(Supporting Information) 化合物 3a $\sim 3 k$ 的 核磁共振氢谱和核磁共振碳谱. 这些材料可以免费从本 刊网站(http://sioc-journal.cn/)上下载.

\section{References}

[1] Badran, N. W. DE 2535228, 1976 [Chem. Abstr. 1976, 85, 5500]. 
[2] Hu, D.-Y.; Xu, J.-T. Chin. J. Med. Guide 2001, 3, 249 (in Chinese). (胡大一, 许俊堂, 中国医药导刊, 2001, 3, 249.)

[3] Kristensen, T. E.; Vestli, K.; Hansen, F. K.; Hansen, T. Eur. J. Org. Chem. 2009, 2009, 5185.

[4] Mei, R.-Q.; Xu, X.-Y.; Li, Y.-C.; Fu, J.-Y.; Huang, Q.-C.; Wang, L.-X.; Mei, R.-Q. Tetrahedron Lett. 2011, 52, 1566.

[5] Alexander, S. K.; Dmitry, E. S.; Albert, G. N.; Alexander, O. C.; Sergei, G. Z. Adv. Synth. Catal. 2012, 354, 3078.

[6] Young, J. L.; Dae, Y. K. Bull. Korean Chem. Soc. 2012, 33, 6.

[7] Dong, J.; Du, D.-M. Org. Biomol. Chem. 2012, 10, 8125.

[8] Alexander, S. K.; Vladislav, G. L.; Alexandr, O. C.; Sergei, G. Z. Eur. J. Org. Chem. 2014, 2014, 3808.

[9] Kim, H.; Yen, C.; Preston, P.; Chin, J. Org. Lett. 2006, 8, 23.

[10] Schoemaker, H. E.; Mink, D.; Wubbolts, M. G. Science 2003, 299, 1694.

[11] Sano, K.; Saito, S.; Hirose, Y.; Kohari, Y.; Nakano, H.; Seki, C.; Tokiwa, M.; Takeshita, M.; Uwai, K. Heterocycles 2013, 87, 6.

[12] Liu, W.-L.; Yang, N.-S.; Chen, Y.-T.; Stephen, L.; Wu, C.-Y.; Lin, C.-H.; Huang, H.-Y. Chem. Eur. J. 2015, 21, 115.

[13] Xie, B.-H.; Guan, Z.; He, Y.-H. J. Chem. Technol. Biotechnol. 2012, $87,1709$.

[14] Guan, Z.; Chen, Y.-L.; Yuan, Y.; Song, J.; Yang, D.-C. PLoS One 2014, 9, e105284.

[15] He, Y.-H.; Song, J.; Yang, D.-C.; Guan, Z. Tetrahedron 2015, 71, 5248 .
[16] Nakajima, N.; Sugimoto, M.; Ishihara, K. J. Mol. Catal. B: Enzym. 2003, 23, 191.

[17] Liu, J.-F.; Wang, X.-Q.; Xu, L.; Zhang, J.-P.; Liang, D.-C.; Chang, W.-R. Chin. Sci. Bull. 2002, 47, 1718 (in Chinese). (刘俊峰, 王新泉, 徐否, 张季平, 梁栋材, 常文瑞, 科学通报, 2002, 47, 1718.)

[18] Zhao, H.-Y.; Jing, T.-Y. J. Biochem. Mol. Biol. 1998, 14, 407 (in Chinese).

(赵虹瑜，静天玉，中国生物化学与分子生物学报，1998，14, 407.)

[19] Luisetti, M.; Piccioni, P. D.; Dyne, K.; Donnini, M.; Bulgheroni, A.; Pasturenzi, L.; Donnetta, A. M.; Peona, V. Int. J. Tissue React. 1991, 4, 187.

[20] Zhang, J.; Zhao, L.-M.; Kang, L.-X. China Surfactant Deterg. Cosmet. 2012, 42, 192 (in Chinese).

(张剑, 赵雷敏, 康林霞, 日用化学工业, 2012, 42, 192.)

[21] Szymczak, R. M.; Mlynarski, J. Tetrahedron: Asymmetry 2014, 25, 813.

[22] Liu, Y.; Kang, T.-R.; Liu, Q.-Z.; Chen, L.-M.; Wang, Y.-C.; Liu, J.; Xie, Y.-M.; Yang, J.-L.; He, L. Org. Lett. 2013, 15, 6090.

[23] Gladkowski, W.; Skrobiszewski, A.; Mazur, M.; Siepka, M.; Pawlak, A.; Mrukowicz, B. O.; Bialonska, A.; Poradowski, D.; Drynda, A.; Urbaniak, M. Tetrahedron 2013, 69, 10414.

[24] Yang, H.-M.; Li, L.; Jiang, K.-Z.; Jiang, J.-X.; Lai, G.-Q.; Xu, L.-W. Tetrahedron 2010, 66, 9708

(Zhao, X.) 